\title{
Stabilization of linear systems with distributed input delay using reduction transformation
}

\author{
CHOON Ki Ahn \\ Faculty of the Department of Automotive Engineering, Seoul National Universty of Science and Technology, Seoul 139-473, Korea
}

Received September 1, 2010; accepted January 10, 2011

\begin{abstract}
We propose a new stabilization method for linear systems with distributed input delay via reduction transformation and Riccati equation approach. In the presented stabilization scheme, the gain matrix of controller is constructed by the well-known linear control technique for delay-free systems. The transformation kernel matrix can be determined by solving the non-symmetric matrix Riccati equation backward with the boundary condition. When point delay systems are considered, it will be shown that the proposed control law degenerates to the standard one for input delay systems.
\end{abstract}

delay systems, delay-free systems, distributed delay, reduction transformation, riccati equation

Citation: Choon K A. Stabilization of linear systems with distributed input delay using reduction transformation. Chinese Sci Bull, 2011, 56: 1413-1416, doi: $10.1007 / \mathrm{s} 11434-010-4152-\mathrm{x}$

In industrial processes, time delays often occur in the transmission of information or material between different parts of a system. The presence of time delays causes serious deterioration on the stability and performance of the system and considerable researches have been done on the control problems of time delay systems [1,2]. Time delay systems are a special case of infinite dimensional systems which have an infinite number of poles. This feature makes the control of time delay systems a difficult task to handle. The easiest way is to reduce time delay systems to delayfree systems and then apply the well developed control techniques for the finite dimensional systems. The Smith predictor may be one of the most well known delay compensation methods utilizing this concept. Although a number of improved versions of the Smith predictor have been proposed by many researchers, the Smith predictor methods suffer from some inherent shortcomings. First, the Smith predictor cannot be applied to unstable systems [3]. The second drawback is that the initial state of the system is ignored in the Smith predictor method. The degradation of the performance is an inevitable result, when it is applied to

email: hironaka@snut.ac.kr the systems with nonzero initial conditions. The third shortcoming is the lack of robustness. The performance of the Smith predictor method is sensitive to the accuracy of the model of the system and time delay. It also suffers from sluggish responses to disturbances.

To overcome the above mentioned difficulties, a number of improved versions of the Smith predictor have been proposed by many researchers [4]. The reduction transformation method is a different approach from the state-space setting to overcome these problems. The explicit reduction transformation method of input delay systems was suggested by Kwon and Pearson [5]. In [5], Kwon and Pearson employed the receding horizon method to input delay systems, where an unexpected good stabilization method (reduction transformation) was founded. The reduction transformation method was extended to the output-feedback case in [6], where a delay-free observer was introduced. This observer can directly estimate the linear function of the state of the system from controls and outputs. This scheme was further developed in [7] to handle systems with unmeasurable disturbances. A rigorous examination of the reduction transformation method can be found in [8], where the scheme was extended to the general 
time-varying systems. Also, the reduction transformation technique was used to design a robust controller for multiple input delayed systems with parametric uncertainties in [9]. The authors in [10] extended the reduction transformation method to linear systems with time-varying input delay. Most results on the reduction transformation were restricted to systems with point delay. To the best of our knowledge, however, for the stabilization of systems with distributed delay via reduction transformation, there is no result in the literature so far, which still remains open and challenging.

Motivated by the above discussion, our main aim in this paper is to shorten this gap by investigating the stabilization problem of linear systems with distributed input delay via reduction transformation. By fixing the transformation kernel matrix as the solution of the non-symmetric Riccati equation, we can transform the input delay system into the delay-free system in spite of distributed delay in the control input. The suggested controller gain matrix can be constructed by using standard linear control methods (for example, pole placement). The transformation kernel matrix can be determined by solving the non-symmetric matrix Riccati equation. If we consider point delay systems, we can obtain the existing result [5] on reduction transformation.

\section{An overview of reduction transformation}

Consider the following input delay system:

$$
\dot{x}(t)=A x(t)+B u(t)+C u(t-\tau),
$$

with initial conditions

$$
x(0)=x_{0}, u(t)=\phi(t), \quad t \in[-\tau, 0],
$$

where $x(t) \in R^{n}$ is the state vector, $u(t) \in R^{m}$ is the control input, $\tau>0$ is the time delay, $A \in R^{n \times n}$, $B \in R^{n \times m}$, and $C \in R^{n \times m}$ are known constant matrices, $x_{0}$ is the initial state vector, and $\phi(t)$ is the initial condition function. The reduction transformation method for the input delay system (1) was suggested by Kwon and Pearson [9]. An extension to time-varying systems can be found in [10]. Consider a linear transformation from $x(t)$ and $u(\alpha)$, $\alpha \in[t-\tau, t]$, to $z(t)$

$$
z(t)=x(t)+\int_{t-\tau}^{t} \mathrm{e}^{A(t-\alpha-\tau)} C u(\alpha) \mathrm{d} \alpha .
$$

Then the system (1) is transformed to

$$
\dot{z}(t)=A z(t)+\left(B+\mathrm{e}^{-A \tau} C\right) u(t),
$$

which is a delay-free system. We consider a linear controller

$$
u(t)=K z(t)
$$

using $z(t)$ as the feedback signal, which is a function of the state $x(t)$ and the past control actions $u(\alpha)$ for $\alpha \in[t-\tau, t]$. The controller gain matrix $K$ can be freely chosen by using any stabilization methods for delay-free systems. If there exists a stabilizing controller (5) for the reduced system (4), then $z(t)$ and $u(t)$ go to zero and, as a result, so does $x(t)$. This indicates that the original system (1) is stable. Similarly, the converse is true. Consequently, using the reduction transformation method, we can obtain a stabilizing controller for the input-delay system (1) by simply designing a stabilizing controller for the ordinary system (4) with the well developed control methods for finite-dimensional systems. Then the stability of the original system (1) is assured by the stability of the transformed system (4).

\section{Main results}

Consider the following linear system with distributed input delay:

$$
\dot{x}(t)=A x(t)+B u(t)+C u(t-\tau)+\int_{0}^{\tau} G(s) u(t-s) \mathrm{d} s,
$$

with initial conditions

$$
x(0)=x_{0}, u(t)=\phi(t), \quad t \in[-\tau, 0],
$$

where $x(t) \in R^{n}$ is the state vector, $u(t) \in R^{m}$ is the control input, $A \in R^{n \times n}, B \in R^{n \times m}$, and $C \in R^{n \times m}$ are known constant matrices, $\tau>0$ is the time delay, $G(s)$ is the $n \times m$ matrix with continuous elements on $[0, \tau]$, and $\phi(t)$ is a continuous function vector. It can be shown that the system (6) can be rewritten as

$$
\dot{x}(t)=A x(t)+B u(t)+C u(t-\tau)+\int_{t-\tau}^{t} G(t-\alpha) u(\alpha) \mathrm{d} \alpha .
$$

Introduce the following linear transformation:

$$
z(t)=x(t)+\int_{t-\tau}^{t} L(t-\alpha) u(\alpha) \mathrm{d} \alpha,
$$

where $L(t-\alpha) \in R^{n \times m}$ is the transformation kernel matrix which is a design function matrix to be determined later. The time derivative of $z(t)$ is given by

$$
\begin{aligned}
\dot{z}(t)=A x(t) & +B u(t)+C u(t-\tau) \\
& +\int_{t-\tau}^{t} G(t-\alpha) u(\alpha) \mathrm{d} \alpha+L(0) u(t) \\
& -L(\tau) u(t-\tau)+\int_{t-\tau}^{t} \frac{\partial L(t-\alpha)}{\partial t} u(\alpha) \mathrm{d} \alpha .
\end{aligned}
$$

Since

$$
x(t)=z(t)-\int_{t-\tau}^{t} L(t-\alpha) u(\alpha) \mathrm{d} \alpha,
$$


we have

$$
\begin{aligned}
\dot{z}(t) & =A\left[z(t)-\int_{t-\tau}^{t} L(t-\alpha) u(\alpha) \mathrm{d} \alpha\right]+B u(t) \\
& +C u(t-\tau)+\int_{t-\tau}^{t} G(t-\alpha) u(\alpha) \mathrm{d} \alpha \\
& +L(0) u(t)-L(\tau) u(t-\tau)+\int_{t-\tau}^{t} \frac{\partial L(t-\alpha)}{\partial t} \times u(\alpha) \mathrm{d} \alpha .
\end{aligned}
$$

Arranging the terms yields

$$
\begin{aligned}
\dot{z}(t) & =A z(t)+(B+L(0)) u(t)+(C-L(\tau)) \\
& \times u(t-\tau)+\int_{t-\tau}^{t}\left[\frac{\partial L(t-\alpha)}{\partial t}-A L(t-\alpha)\right. \\
& +G(t-\alpha)] u(\alpha) \mathrm{d} \alpha .
\end{aligned}
$$

If the transformation kernel matrix $L(t-\alpha)$ is selected as

$$
\begin{gathered}
\frac{\partial L(t-\alpha)}{\partial t}=A L(t-\alpha)-G(t-\alpha), \\
L(\tau)=C, \quad \alpha \in[t, t-\tau],
\end{gathered}
$$

then the original distributed delay system eq. (6) can be transformed to the following delay-free system:

$$
\dot{z}(t)=A z(t)+(B+L(0)) u(t) .
$$

If we have the solution of the differential eq. (14) with the boundary condition (15), the existing linear control methods can be applied to the transformed delay-free system (16). Now we are ready to state the stabilization result for linear systems with distributed input delay using reduction transformation.

Theorem 1 Assume that the pair $(A, B+L(0))$ is stabilizable. If we apply the following control input:

$$
u(t)=K\left[x(t)+\int_{t-\tau}^{t} L(t-\alpha) u(\alpha) \mathrm{d} \alpha\right]
$$

where

(1) $K$ is selected to make $A z(t)+(B+L(0)) K$ Hurwits matrix

(2) $L(\cdot)$ is obtained by solving the following nonsymmetric Riccati equation:

$$
\dot{L}(t)-A L(t)+G(t)=0, \quad t \in[0, \tau],
$$

with the boundary condition $L(\tau)=C$, then the original delay system (6) can be stabilized.

Proof Since the transformed system eq. (16) is a delay-free system, we can apply a linear control method for finite dimensional systems. Let the control input $u(t)$ be $K z(t)$. From eq. (16), we have

$$
\dot{z}(t)=[A+(B+L(0)) K] z(t) .
$$

If $K$ is selected to make $A z(t)+(B+L(0)) K$ Hurwits matrix, we have $z(t) \rightarrow 0$. Since $u(t)=K z(t)$, it is clear that $u(t) \rightarrow 0$. From (9), we can conclude that $x(t) \rightarrow 0$. This completes the proof.

If we consider point delay systems, i.e. $G(\cdot)=0$, the standard result [9] on reduction transformation can be obtained.

Corollary 1 If we consider the following point delay system

$$
\dot{x}(t)=A x(t)+B u(t)+C u(t-\tau)
$$

then the result proposed in Theorem 1 degenerates to the standard stabilization result [9].

Proof Since we consider the point delay system, let $G(\cdot)=0$. Then $(18)$ becomes

$$
\dot{L}(t)-A L(t)=0, \quad t \in[0, \tau],
$$

with the boundary condition $L(\tau)=C$. The definite solution of this differential equation is

$$
L(t)=\mathrm{e}^{A t} K_{1},
$$

where $K_{1}$ is a constant matrix. With the information of the boundary condition $L(\tau)=C$, the constant matrix $K_{1}$ can be determined as

$$
K_{1}=\mathrm{e}^{-A \tau} C
$$

Thus, we have the following transformation kernel matrix:

$$
L(t)=\mathrm{e}^{A(t-\tau)} C .
$$

In this case, the reduction transformation (9) becomes

$$
z(t)=x(t)+\int_{t-\tau}^{t} \mathrm{e}^{A(t-\alpha-\tau)} C u(\alpha) \mathrm{d} \alpha
$$

and the transformed delay-free system is given by

$$
\dot{z}(t)=A z(t)+\left(B+\mathrm{e}^{-A \tau} C\right) u(t) .
$$

This result is equivalent to the standard stabilization result proposed in [9]. This completes the proof.

The stabilization method of this paper can be summarized as follows:

(i) With the information of the system matrices $A$ and $G(\cdot)$, solve the following non-symmetric Riccati equation backward from $\tau$ to 0 with the boundary condition $L(\tau)=C:$

$$
\dot{L}(t)-A L(t)+G(t)=0, \quad t \in[0, \tau],
$$

and store $L(\cdot)$.

(ii) Determine $K$ which makes $A z(t)+(B+L(0)) K$ Hurwits matrix.

(iii) Use the following control input with the predetermined matrices $K$ and $L(\cdot)$ : 


$$
u(t)=K\left[x(t)+\int_{t-\tau}^{t} L(t-\alpha) u(\alpha) \mathrm{d} \alpha\right]
$$

Remark 1 Since eq. (18) is a special case of nonsymmetric matrix Riccati equations, we can obtain the solution of eq. (18) using results presented in $[11,13]$.

Remark 2 Since the transformed system eq. (16) is a delay-free ordinary system, we can apply various kinds of linear control methods to the transformed system. In this paper, we used the typical pole-placement technique for simplicity.

\section{Conclusions}

In this paper, a new stabilization method is presented for linear systems with distributed input delay via reduction transformation and Riccati equation approach. The original system with distributed input delay can be transformed into the delay-free system by fixing the transformation kernel matrix as the solution of the nonsymmetric Riccati equation. The feature of our control scheme is that the controller gain matrix $K$ is selected by using typical linear control technique and the transformation kernel matrix $L(\cdot)$ can be constructed by solving the nonsymmetric matrix Riccati equation with the boundary condition. The result presented in this paper degenerates to the standard stabilization result when we consider point delay systems.

1 Marshall J E. Control of Time-delay Systems. Stevenage: Peter Perigrinius, 1997

2 Malek-Zavarei M, Jamshidi M. Time-delay Systems Analysis, Optimization and Applications. Amsterdam: Elsevier, 1987

3 Furukawa T, Shimemura E. Predctive control for systems with time delay. Int J Control, 1983, 37: 399-412

4 Whalley R, Zeng Z..A robust smith predictor. Trans IChemE, 1996,74 A: 21-27

5 Kwon W H, Pearson A E. Feedback stabilization of linear systems with delayed control. IEEE Trans Automat Contr, 1980, 25: 266-269

6 Watanabe K, Ito M. An observer for linear feedback control laws of multivariable systems with multiple delays in controls and outputs. Sys Contr Lett, 1981: 1: 54-59

7 Watanabe K, Sato M. A process-model control for multivariable systems with multiple delays in inputs and outputs subject to unmeasurable disturbances. Int J Contr, 1984, 39: 1-17

8 Arstein Z. Linear systems with delayed control: A reduction. IEEE Trans Automat Contr, 1982, 27: 869-879

9 Moon Y S, Park P G, Kwon W H. Robust stabilization of uncertain input delayed systems using reduction method. Automatica, 2001, 37: 307-312

10 Yue D, Han Q L. Delayed feedback control of uncertain systems with time-varying input delay. Automatica, 2005, 41: 233-240

11 Telford J, Moore J B. On the existence of solutions to nonsymmetric algebraic riccati equations. Lecture Notes of the Workshop on "The Riccati Equation in Control, Systems and Signal", 1989: 83-86

12 Jodar L, Cortes Lopez J C Rational matrix approximation with a priori error bounds for non-symmetric matrix riccati equations with analytic coefficients. IMA J Num Analys, 1998, 18: 545-561

13 Jank G, Freiling G, Abou-Kandil H. A review of nonsymmetric riccati equations. In: Proc MTNS St Luis-USA, 1996, 83-86

Open Access This article is distributed under the terms of the Creative Commons Attribution License which permits any use, distribution, and reproduction in any medium, provided the original author(s) and source are credited. 